Check for updates

Cite this: RSC Adv., 2018, 8, 23817

Received 31st March 2018

Accepted 6th June 2018

DOI: 10.1039/c8ra02791a

rsc.li/rsc-advances

\section{Flowery nickel-cobalt hydroxide via a solid-liquid sulphur ion grafting route and its application in hybrid supercapacitive storage $\uparrow$}

\begin{abstract}
Lin Ye, Zepei Bao, $\$$ Yuguang Zhao and Lijun Zhao (D) *
In our research, a two-step solid-liquid route was employed to fabricate flowery nickel-cobalt hydroxide with sulphur ion grafting (Ni1CO2-S). The utilization of $\mathrm{NaOH} /$ agar and $\mathrm{Na}_{2} \mathrm{~S}$ /agar could efficiently retard the release rates of $\mathrm{OH}^{-}$or $\mathrm{S}^{2-}$ ions at the solid-liquid interface due to strong bonding between agar hydrogel and these anions. Ni1Co2-S generally displays ultrathin flowery micro-frame, ultrathin internal nanosheets and expanded pore size. Besides, the introduction of suitable sulphide species into nickelcobalt hydroxide could improve its conductivity due to the lower band gap of $\mathrm{Ni}-\mathrm{Co}$ sulphide. The supercapacitive electrode Ni1Co2-S presented capacitance of $1317.8 \mathrm{~F} \mathrm{~g}^{-1}$ (at $1 \mathrm{~A} \mathrm{~g}^{-1}$ ) and suitable rate performance (77.9\% at $10 \mathrm{~A} \mathrm{~g}^{-1}$ and $59.3 \%$ at $20 \mathrm{~A} \mathrm{~g}^{-1}$ ). Furthermore, a hybrid supercapacitor (HSC) was developed utilizing positive Ni1Co2-S and negative activated carbon electrodes. As expected, the HSC device exhibited excellent specific capacitance (117.1 $\mathrm{F} \mathrm{g}^{-1}$ at $1 \mathrm{~A} \mathrm{~g}^{-1}$ ), considerable energy densities (46.7 $\mathrm{W} \mathrm{h} \mathrm{kg}^{-1}$ at $0.845 \mathrm{~kW} \mathrm{~kg}^{-1}$ and $27.5 \mathrm{~W} \mathrm{~h} \mathrm{~kg}^{-1}$ even at $9 \mathrm{~kW} \mathrm{~kg}^{-1}$ ) and suitable cycling performance, which further illuminated the high energy storage capacity of Ni1Co2-S.
\end{abstract}

\section{Introduction}

Numerous electronic equipments such as electric vehicles, consumer electronics and portable devices have facilitated our daily lives, but some adverse issues such as low recyclability and energy storage capacity remain for their extensive applications. Therefore, substantial attention is focused on achieving efficient energy storage of supercapacitors (SCs) and their successful applications in various electronic equipments due to their high power deliveries, long lifespans and rapid charge/ discharge rates. ${ }^{1-4}$ To fully promote energy density and enlarge the potential window, assembling hybrid SCs is a feasible strategy, wherein a redox electrode serves as the energy source and an electrical double layer electrode serves as the power source. ${ }^{5,6}$ Meanwhile, exploring efficient functional electrode materials plays a vital role for integrated performance of HSCs. As typical battery-type materials, ${ }^{7-9}$ a class of $\mathrm{Ni} / \mathrm{Co}$ hydroxides represent multiple oxidation states, high theoretical values and long discharge platforms ${ }^{, 0-12}$ which endow them with high potentials in supercapacitive materials.

The actual capacitance values of electrode materials are always lower than the theoretical values due to many factors

Key Laboratory of Automobile Materials, Ministry of Education and School of Materials Science and Engineering, Jilin University, Changchun, 130022, P. R. China.E-mail: lijunzhao@jlu.edu.cn

$\dagger$ Electronic supplementary information (ESI) available. See DOI: 10.1039/c8ra02791a

\$ These authors contributed equally to this work. such as specific surface area, porosity, conductivity, crystal structure and chemical composition. ${ }^{13-16}$ Thus, a rational structural design is highly desired to further improve electrochemical performances, especially while fabricating multi-level structures with large specific areas and optimizing components. On the one hand, versatile micro/nanoflower structures have been constructed and applied in catalysts, ${ }^{17}$ batteries, ${ }^{18}$ and supercapactors. ${ }^{19}$ Sufficient interior space and large specific surface area expose more active areas and shorten contact distance and thus, the generation of ultrathin nanosheets in flowery structures enhances electrochemical performance. On the other hand, the conductivity or stability performance of $\mathrm{Ni}$ / Co hydroxides needs further improvement..$^{20-22}$ Recently, many studies have reported different forms of S incorporated with $\mathrm{Ni} /$ Co hydroxides, because Ni/Co sulphides display lower band gap values than their hydroxides. ${ }^{23-27}$ For instance, Zhou and coworkers reported a distinct core-shell $\mathrm{Ni}_{3} \mathrm{~S}_{2}$ nanorod@ $\mathrm{Ni}(\mathrm{OH})_{2}$ nanosheet nanoarchitecture that presented an enhanced performance of $1037.5 \mathrm{~F} \mathrm{~g}^{-1}$ (at $5.1 \mathrm{Ag}^{-1}$ ) than monocomponent $\mathrm{Ni}(\mathrm{OH})_{2}$, because the hybrid structure-integrated $\mathrm{Ni}(\mathrm{OH})_{2}$ nanosheets with rich active sites and crystalline $\mathrm{Ni}_{3} \mathrm{~S}_{2}$ nanorods promoted rapid electron transfer. ${ }^{23}$ Also, core-shell $\mathrm{NiCo}_{2} \mathrm{~S}_{4} @ \mathrm{Co}(\mathrm{OH})_{2}$ nanotube arrays were prepared, which showed lower polarization and improved reactivity compared to bare $\mathrm{NiCO}_{2} \mathrm{~S}_{4}$ structures. ${ }^{24}$ Recently, a gas-solid reaction route was selected to fabricate novel metallic nickel hydroxide nanosheets utilizing $\mathrm{H}_{2} \mathrm{~S}$ gas to treat $\beta-\mathrm{Ni}(\mathrm{OH})_{2}$ nanosheets at $110^{\circ} \mathrm{C}$. The introduction of sulphur into $\mathrm{Ni}(\mathrm{OH})_{2}$ efficiently promoted electrical conductivity, and the material exhibited metallic 
property. ${ }^{25}$ Therefore, slight sulphur ion grafting route has been designed to improve the conductivities of hydroxide samples.

For the synthesis of electrode materials, various methods such as the microwave method, ${ }^{28}$ solvothermal/hydrothermal method, ${ }^{29,30}$ electro-deposition reaction ${ }^{31}$ and calcination treatment ${ }^{32}$ have been reported. In spite of great improvements in the energy conversion of SCs, high costs and unsuitable synthesis processes hinder their further applications. Up to now, few studies have been reported on the $\mathrm{NaOH} /$ agar and $\mathrm{Na}_{2} \mathrm{~S}$ /agar hydrogel synthetic method. Herein, we used a twostep solid-liquid method to obtain $\mathrm{Ni}-\mathrm{Co}$ hydroxide and $\mathrm{Ni}-$ Co hydroxide with $\mathrm{S}^{2-}$ grating. The distinct agar hydrogel method has the following advantages: (1) the preparation of agar hydrogel only needs a short heat treatment time of $10 \mathrm{~min}$, and the subsequent reactions are carried out at room temperature, which saves energy consumption; (2) there is controllable ionic diffusion in the solid-liquid interface owing to strong bonding between hydrogel and anions $\left(\mathrm{OH}^{-}\right.$or $\mathrm{S}^{2-}$ ions); (3) facile reaction equipments scale up sample preparation. In the hydrogel-assisted reaction, the flowery nickel-cobalt hydroxide via sulphur ions grafting not only introduces small amount of sulphide species, but also expands the pore size, which greatly stimulates charge transport. As anticipated, the Ni1Co2-S electrode and the corresponding hybrid supercapacitor (HSC) device deliver excellent capacitances, appropriate rate performances and cycling stabilities.

\section{Experimental section}

\subsection{Preparation of $\mathrm{NaOH} /$ agar and $\mathrm{Na}_{2} \mathrm{~S}$ /agar hydrogel}

First, 12 mmol sodium hydroxide $(\mathrm{NaOH})$ and $0.5 \mathrm{~g}$ agar powder were transferred to a $100 \mathrm{~mL}$ beaker. Then, $10 \mathrm{~mL}$ deionized water was added to the beaker. The mixture was homogeneously dispersed via consistent ultrasonication and then heated in a water bath at a temperature of $85{ }^{\circ} \mathrm{C}$ for $10 \mathrm{~min}$. The obtained mixture was placed at room temperature to form $\mathrm{NaOH} /$ agar hydrogel. Similarly, $0.7 \mathrm{~g}$ agar was added to $20 \mathrm{~mL}$ $0.03 \mathrm{~mol} \mathrm{~L}^{-1}$ sodium sulphide $\left(\mathrm{Na}_{2} \mathrm{~S}\right)$ aqueous solution, and the mixture was homogeneously dispersed, heated at $85{ }^{\circ} \mathrm{C}$ for $10 \mathrm{~min}$ and cooled to room temperature. The obtained hydrogel was marked as $\mathrm{Na}_{2} \mathrm{~S} /$ agar hydrogel.

\subsection{Preparation of Ni1Co2, Ni1Co2-AN, Ni2Co1, Co3 and Ni3}

First, $1.5 \mathrm{mmol}$ nickel nitrate hexahydrate $\left(\mathrm{Ni}\left(\mathrm{NO}_{3}\right)_{2} \cdot 6 \mathrm{H}_{2} \mathrm{O}\right)$, $3 \mathrm{mmol}$ cobalt hexahydrate $\left(\mathrm{Co}\left(\mathrm{NO}_{3}\right)_{2} \cdot 6 \mathrm{H}_{2} \mathrm{O}\right)$ and $1.5 \mathrm{mmol}$ ammonium chloride $\left(\mathrm{NH}_{4} \mathrm{Cl}\right)$ were added to a solution containing $15 \mathrm{~mL}$ deionized water and $50 \mathrm{~mL} N, N$-dimethyl formamide (DMF). The mixture was stirred for several minutes and gradually dropped on $\mathrm{NaOH} /$ agar hydrogel. After that, the beaker was placed for $24 \mathrm{~h}$ in a dark place without any heat treatment. The precipitate generated was collected, washed using abundant water and ethanol, and heated to dry. The above-mentioned sample was denoted as Ni1Co2. In the absence of $\mathrm{NH}_{4} \mathrm{Cl}$, the obtained precipitate was denoted as Ni1Co2-AN. Similarly, the molar ratios of $\mathrm{Ni}\left(\mathrm{NO}_{3}\right)_{2} \cdot 6 \mathrm{H}_{2} \mathrm{O}$ and $\mathrm{Co}\left(\mathrm{NO}_{3}\right)_{2} \cdot 6 \mathrm{H}_{2} \mathrm{O}$ were adjusted from $3: 0$,
$2: 1$ and $0: 3$, and the other conditions were not changed; hence, the corresponding precipitates were marked as Ni3, Ni2Co1 and Co3.

\subsection{Preparation of Ni1Co2-S, Ni1Co2-AN-S, Ni2Co1-S, Ni3-S and Co3-S}

First, $50 \mathrm{mg}$ Ni1Co2 precursor was dispersed in $40 \mathrm{~mL}$ deionized water with ultrasonication to form Ni1Co2 suspension. The above-mentioned suspension was dropped on $\mathrm{Na}_{2} \mathrm{~S} / \mathrm{agar}$ hydrogel and reacted at room temperature for $24 \mathrm{~h}$. The precipitate generated was collected, washed using abundant water and ethanol, and heated to dry. The as-obtained composite was marked as Ni1Co2-S. The Ni1Co2 precursor was converted to Ni1Co2-AN, Ni2Co1, Ni3 and Co3 in turn; the samples obtained were denoted as Ni1Co2-AN-S, Ni2Co1-S, Ni3-S and Co3-S, respectively.

\subsection{General characterization}

On the basis of frequently used characterization technologies such as field emission scanning electron microscopy (FESEM, JEOL6700F) and transmission electron microscopy (TEM, JEM2100F), superficial microtopographies and internal structures of our samples were studied in detail. Besides, an accelerating voltage of $200 \mathrm{kV}$ was employed in the selected area electron diffraction (SAED) measurement. Wide angle X-ray diffraction (XRD, D8 ADVANCE) was also employed to analyze the phase components of our samples utilizing $\mathrm{Cu}-\mathrm{K} \alpha$ radiation with a scanning range from $10^{\circ}$ to $80^{\circ}$. Furthermore, X-ray photoelectron spectroscopy (XPS, ESCAlab220i-XL, Al-K $\alpha$ source) and Raman microspectrometry (Renishaw, inVia-Reflex, $532 \mathrm{~nm}$ ) tests were performed to gain insights into the valence states of different elements and chemical bonds of our samples. Furthermore, inductively coupled plasma-optical emission spectrometry (ICP-OES, SPECTRO CIROS VISION) was used to analyze the accurate mole ratio of metal anions. Fourier transform infrared (FTIR) spectrometry was carried out to study the chemical bonds in our samples. For obtaining the Barrett-Joyner-Halenda (BJH) mode, a Micromeritics ASAP-2020M apparatus was operated.

\subsection{Electrode preparation and electrochemical measurements}

The preparatory method of positive electrodes based on $\mathrm{Ni}-\mathrm{Co}$ nanocomposites was as follows: first, we ground active materials poly(vinylidene fluoride) (PVDF) and acetylene black (AB) in a mass ratio of $85: 5: 10$ for several minutes. The mixture was added to a suitable amount of $\mathrm{N}$-methyl-2-pyrrolidone (NMP) solvent and stirred constantly to generate a uniformly distributed ropy slurry. Then, the ropy slurry was uniformly applied on a porous $\mathrm{Ni}$ foam (effective area of $1 \mathrm{~cm}^{2}$ was controlled) and dried at $110{ }^{\circ} \mathrm{C}$ for $6 \mathrm{~h}$ in a vacuum oven. A series of electrodes were prepared including Ni1Co2, Ni1Co2-AN, Ni2Co1, Ni3, Co3, Ni1Co2-S, Ni1Co2-AN-S, Ni2Co1-S, Ni3-S and Co3-S. For effective comparison, we controlled the mass loadings of the as-mentioned electrodes to $1.2 \pm 0.2 \mathrm{mg} \mathrm{cm}^{-2}$. An active carbon (AC) electrode was also prepared utilizing 
a similar method except that we changed the mass ratio of the mixture (AC : PVDF : $\mathrm{AB}=90: 5: 5$ ). The mass loading of the AC electrode was controlled in the range of $2.9 \pm 0.1 \mathrm{mg} \mathrm{cm}^{-2}$. In the Ni1Co2//AC HSC device, we utilized an Ni1Co2 electrode as the positive electrode and AC positive electrode as the negative electrode. The total mass loading of the Ni1Co2-S//AC device was controlled in the range $4.1 \pm 0.3 \mathrm{mg}$.

\section{Results and discussion}

\subsection{Characterization of morphology, microstructure and composition}

The overall fabrication procedure of flowery Ni1Co2 precursor is displayed in Fig. 1. $\mathrm{OH}^{-}$ions were slowly transferred through the solid-liquid interface due to strong bonding between $\mathrm{OH}^{-}$ ions and agar hydrogel. These $\mathrm{OH}^{-}$ions were slowly incorporated with $\mathrm{Ni}^{2+}$ and $\mathrm{Co}^{2+}$ ions, thus generating binary $\alpha-(\mathrm{Ni}-\mathrm{Co})$ hydroxide. After that, the $\mathrm{Na}_{2} \mathrm{~S} /$ agar hydrogel slowly provided $\mathrm{S}^{2-}$ ions for partial incorporation with metal ions from nickelcobalt hydroxide. A small amount of sulphur ions was grafted on Ni1Co2 hydroxide (Ni1Co2-S).

As shown in Fig. 2a and S1a and b, $\dagger$ Ni1Co2 displayed wellproportioned flowery clusters (diameter up to several micrometers) and ultrathin internal nanosheets. In comparison, the Ni1Co2-AN sample showed irregular nanoflowers with the coexistence of small sheets and large sheets (in Fig. S2a and $b \dagger$ ), because there was no chelation between $\mathrm{NH}_{4}{ }^{+}$and $\mathrm{OH}^{-}$ions. Furthermore, the mole ratio of $\mathrm{Ni}\left(\mathrm{NO}_{3}\right)_{2}: \mathrm{Co}\left(\mathrm{NO}_{3}\right)_{2}$ was adjusted from $3: 0,2: 1$ to $0: 3$ to optimize the proportion. Staked nanoflowers with insufficient space (Ni2Co1 or Co3) and conglobated nanoparticles (Ni3) are exhibited in Fig. S2c-h, $\uparrow$ indicating that various mole ratios of metal nitrates could significantly influence the ionic reaction and tune the microtopography. On the basis of the accurate ICP-OES data in Table $\mathrm{S} 1, \dagger$ the $\mathrm{Ni}$ : Co ratios of Ni1Co2, Ni1Co2-AN, Ni3, Ni2Co1 and Co3 were $1: 2.27,1: 2.26,3: 0,1.86: 1$ and $3: 0$, respectively, which matched the dosages of metal nitrates, and this indicated a uniform co-precipitation reaction.

Subsequently, we utilized $\mathrm{Na}_{2} \mathrm{~S} /$ agar hydrogel to grate $\mathrm{S}^{2-}$ ions on Ni1Co2 and obtain Ni1Co2-S. The lower solubilities of metal sulphides than those of hydroxides caused ion exchanges between $\mathrm{OH}^{-}$and $\mathrm{S}^{2-}$ ions. Fig. $\mathrm{S} 3 \uparrow$ reveals the colour change from green (Ni1Co2) to grey green (Ni1Co2-S), which supported the occurrence of $\mathrm{S}^{2-}$ ion grating processes. In Fig. $2 \mathrm{~b}$ and $\mathrm{S} 1 \mathrm{c}$

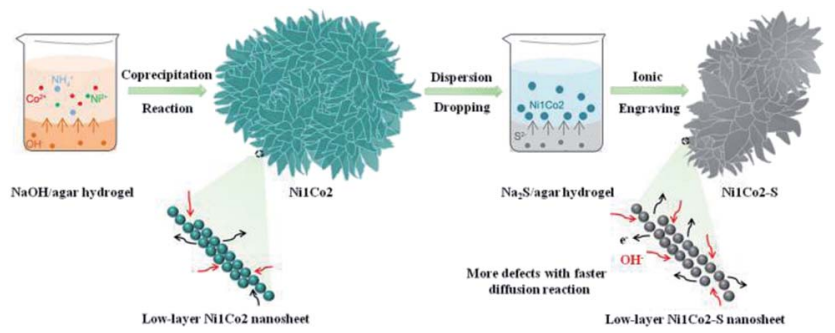

Fig. 1 Schematic exhibition of the fabrication procedure of flowery Ni1Co2-S

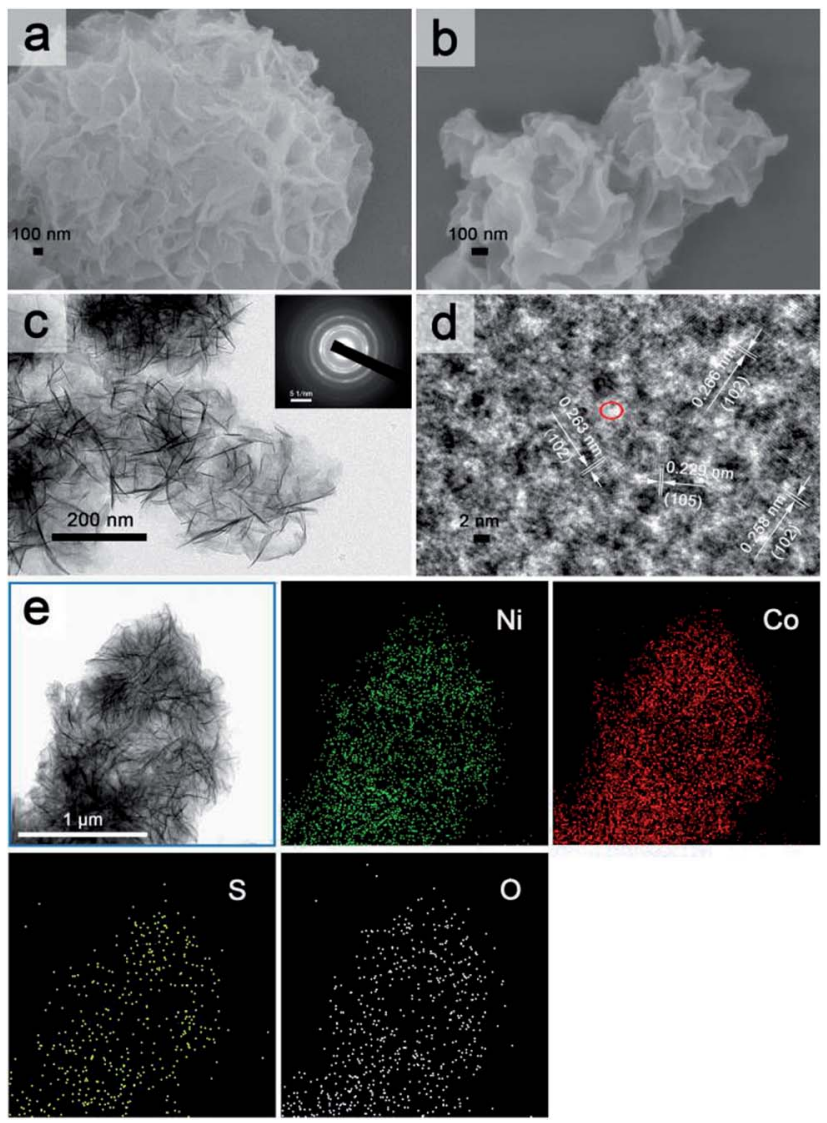

Fig. 2 FESEM images of (a) Ni1Co2 and (b) Ni1Co2-S; (c) TEM image of Ni1Co2-S with inset of SEDA image; (d) HETEM image of Ni1Co2-S; (e) TEM figure and corresponding elemental mapping images for Ni1Co2-S

and $d, \dagger$ we can see that the pore sizes of Ni1Co2-S extended with the partial damage of pristine flowery frames. Furthermore, the BJH modes of Ni1Co2 and Ni1Co2-S were also obtained to study the details of pore size distribution. As shown in Fig. $\mathrm{S} 4, \dagger$ the main pore size of $\mathrm{Ni1Co} 2$ was concentrated in a narrow range from 1.7 to $7.5 \mathrm{~nm}$, whereas that of Ni1Co2-S exhibited a wider range from 1.7 to $50 \mathrm{~nm}$. This clear broadening of pore size further revealed a structural change after $\mathrm{S}^{2-}$ ion grating. Fig. 2c displays the transparent structure of Ni1Co2-S, and Fig. S5† presents nanosheet thicknesses (mainly ranging from 2.2 to $5.3 \mathrm{~nm}$ ), and these results revealed the ultrathin property of Ni1Co2-S. In the inset of Fig. $2 c$, the diffraction circles reveal the polycrystalline property of Ni1Co2S. In Fig. 2d, the lattice fringes of $0.258,0.263$ and $0.266 \mathrm{~nm}$ resulted from the (102) planes of nickel-cobalt hydroxide. Notably, many internal pores and defects could be observed in Fig. $2 \mathrm{~d}$ such as the red elliptical area, which probably could be ascribed to the grating effect of $\mathrm{S}^{2-}$ ion. Furthermore, the elemental mapping of Ni1Co2-S is provided to illuminate element distribution. As depicted in Fig. 2e, Ni, Co, S and $\mathrm{O}$ uniformly existed in the flowery Ni1Co2-S architecture. Based on energy dispersive spectrometry (EDS) data shown in Fig. S6, $\dagger$ the mole ratio $\mathrm{S}: \mathrm{O}$ was found to be $1: 5.14$, indicating that 


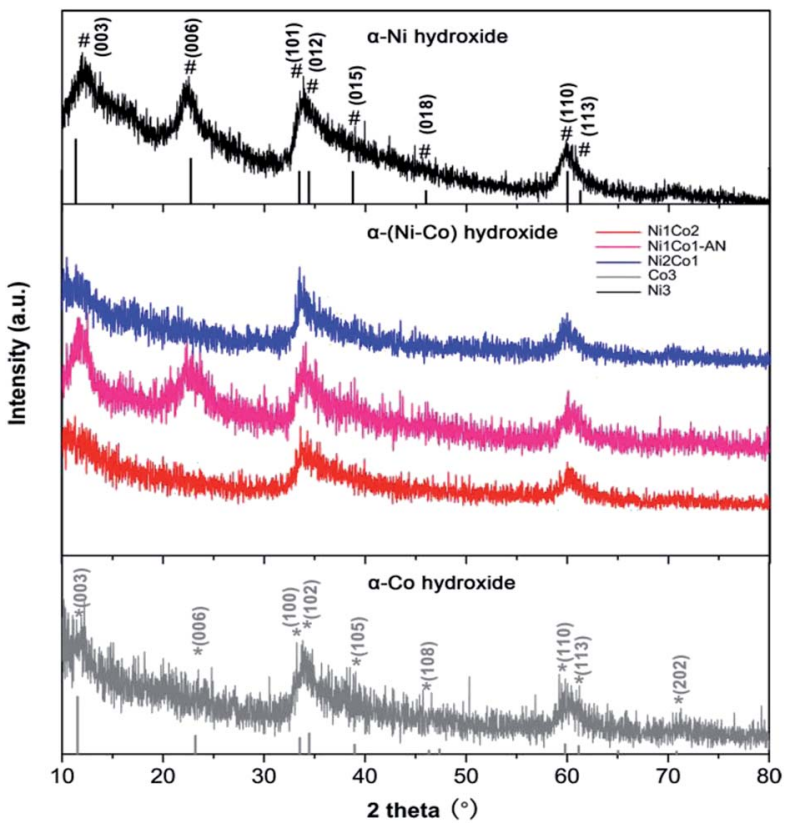

Fig. 3 XRD patterns of Ni1Co2, Ni1Co2-AN, Ni2Co1, Co3 and Ni3 samples.

Ni1Co2-S was composed of Ni-Co hydroxides with slight $\mathrm{S}^{2-}$ grating. Also, the mole ratio of $\mathrm{Ni}$ : Co reached $1: 1.89$, which also generally coincided with the ratio of $1: 2$ (dosage of metal nitrates).

In Fig. 3, the main peaks of $\mathrm{Ni} 3$ are directly indexed to $\alpha$-Ni hydroxide with turbostratic disorder, and the peaks of $\mathrm{Co} 3$ also present the property of $\alpha$-Co hydroxide. ${ }^{33,34}$ Meanwhile, the peaks of Ni1Co2, Ni1Co2-AN and Ni2Co1 are analogous with those of Ni3 and Co3, indicating the formation of binary $\alpha-(\mathrm{Ni}-$ Co) hydroxide. To further study the structures of these hydroxides, their FTIR patterns are displayed in Fig. S7a. $\dagger$ In samples of Ni1Co2, Ni1Co2-AN, Ni2Co1, Ni3 and Co3, a series of broad peaks centred at $3400-3500 \mathrm{~cm}^{-1}$ were assigned to the hydrogen bonding with intercalated water molecules. Besides, the peaks at $1000-1500 \mathrm{~cm}^{-1}$ were ascribed to intercalated $\mathrm{NO}_{3}{ }^{-}$ions, and the peaks at $400-700 \mathrm{~cm}^{-1}$ resulted from $\mathrm{M}(\mathrm{Ni} / \mathrm{Co})-\mathrm{O}-\mathrm{H}$ bending and $\mathrm{M}-\mathrm{O}$ stretching vibrations. These FTIR results demonstrated the $\alpha$-hydroxide features of Ni1Co2, Ni1Co2-AN, Ni2Co1, Ni3 and Co3. ${ }^{33-35}$ Furthermore, the Raman spectra of these samples supported the chemical bonds of hydroxide precursors. In Fig. S8, $\uparrow$ the Raman peaks of $\mathrm{Co} 3$ located at 184.6, 464.0, 507.6, 599.8 and $666.2 \mathrm{~cm}^{-1}$ coincided with the features of $\alpha-\mathrm{Co}(\mathrm{OH})_{2} \cdot{ }^{36-38}$ In the Raman pattern of Ni3, the peaks at $304.2,400-600$, and $1093.9 \mathrm{~cm}^{-1}$ corresponded to $\mathrm{Ni}-\mathrm{O}$ stretching, E-type vibration of the $\mathrm{Ni}-\mathrm{OH}$ lattice and hydroxyl group vibration mode of the $\mathrm{Ni}(\mathrm{OH})_{2}$ phase. $^{39,40}$ In contrast, Ni1Co2, Ni1Co2-AN and Ni2Co1 maintained the peak locations of Ni3 and $\mathrm{Co} 3$ with some peak shifts, indicating the existence of binary Ni-Co hydroxide. Therefore, we successfully fabricated a series of unitary $\alpha-(\mathrm{Ni} / \mathrm{Co})$ or binary Ni-Co hydroxides.

In the XRD pattern shown in Fig. 4a, Ni1Co2-S displays similar peaks to Ni1Co2, demonstrating that Ni1Co2-S mainly
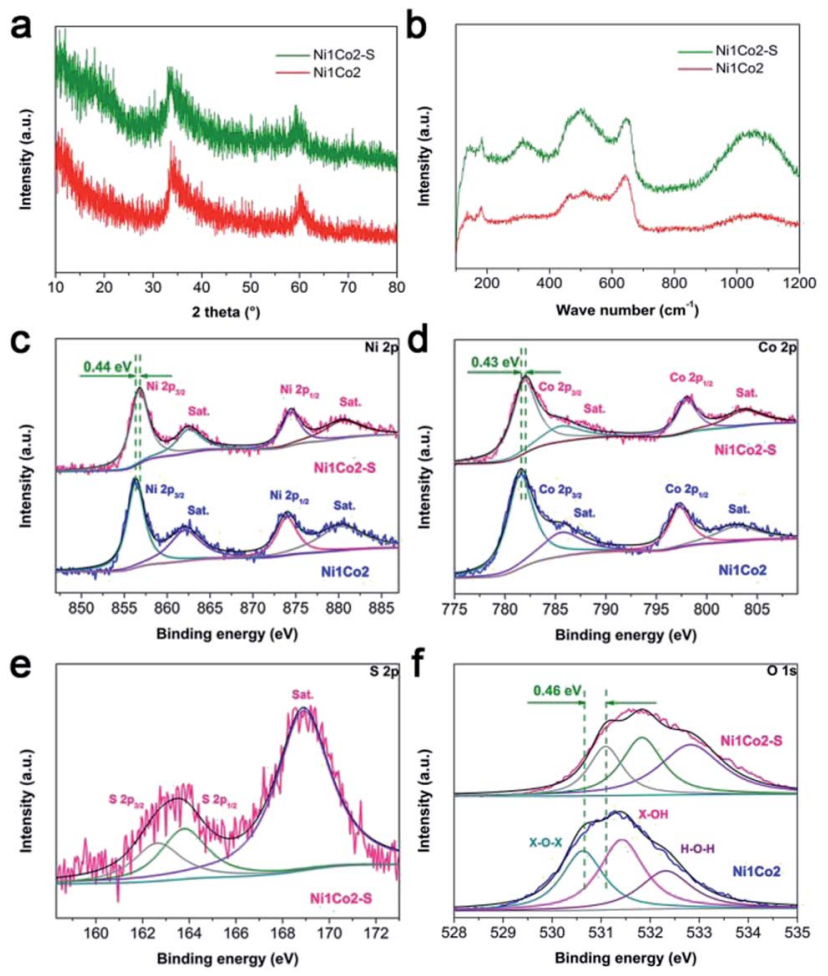

Fig. 4 (a) XRD patterns of Ni1Co2 and Ni1Co2-S samples; (b) Raman patterns of Ni1Co2 and Ni1Co2-S samples; (c) Ni 2p, (d) Co 2p, (e) S 2p and (f) $\mathrm{O} 1 \mathrm{~s} X \mathrm{XS}$ spectra of $\mathrm{Ni1CO} 2$ and Ni1Co2-S samples. Herein, $\mathrm{X}$ in (f) represents $\mathrm{Ni}$ or Co metal element.

contains $\alpha$-(Ni-Co) hydroxide. Compared to peaks from Ni1Co2, a clear negative shift $\left(c a .0 .6\right.$ to $\left.0.9^{\circ}\right)$ can be observed in the XRD pattern of Ni1Co2-S because the slight $\mathrm{S}^{2-}$ grating reaction extends the internal planar spacing and pore size. No clear signals of Ni-Co sulphide can be detected because of weak crystallinity and low content of sulphide at room-temperature. By comparing the FTIR and Raman patterns of Ni1Co2 and Ni1Co2-S (in Fig. S7b and $4 \mathrm{~b} \dagger)$, we further ascertain the $\alpha-(\mathrm{Ni}-$ Co) hydroxide component in Ni1Co2-S.

We utilized the XPS patterns of Ni1Co2 and Ni1Co2-S to evaluate the variation before and after $\mathrm{S}^{2-}$ grafting. All spectra were calibrated based on the C $1 \mathrm{~s}$ benchmark of $284.6 \mathrm{eV}$. In the Ni 2p spectra of Ni1Co2 (in Fig. 4c), the peaks at 856.33 and $873.95 \mathrm{eV}$ corresponded to $\mathrm{Ni} 2 \mathrm{p}_{3 / 2}$ and $2 \mathrm{p}_{1 / 2}$ with a separation of $17.62 \mathrm{eV}$, which was in line with the feature of the $\mathrm{Ni}(\mathrm{OH})_{2}$ phase. ${ }^{41,42}$ In Fig. $4 \mathrm{~d}$, Ni1Co2 displays Co $2 \mathrm{p}_{3 / 2}$ and $2 \mathrm{p}_{1 / 2}$ peaks (at 781.51 and $797.29 \mathrm{eV}$ ) with a separation of $15.78 \mathrm{eV}$, indicating the co-existence of divalent and trivalent states of Co. ${ }^{43,44}$ The divalent and trivalent states of Co co-existed in $\alpha-(\mathrm{Ni}-\mathrm{Co})$ hydroxides. For Ni1Co2-S, the energy-bond separations of Ni 2p and Co $2 p$ levels were 17.67 and $16.05 \mathrm{eV}$, respectively, verifying slight reduction of partial cobalt ions during the ion exchange process. In Fig. 4e, the peaks at 162.63 and $163.77 \mathrm{eV}$ represented $\mathrm{S} 2 \mathrm{p}_{3 / 2}$ and $2 \mathrm{p}_{1 / 2}$ levels, respectively, and a satellite peak at $168.89 \mathrm{eV}$ was ascribed to $\mathrm{S}_{4} \mathrm{O}_{6}{ }^{2-}$ ions with high oxidation states on the surface. Similar S $2 p$ spectra could be found in many reported nickel/cobalt sulfides, ${ }^{45-47}$ which suggested 
successful grating of $\mathrm{S}^{2-}$ ions in Ni1Co2-S. In contrast to the observations for Ni1Co2, Ni 2p, Co 2p and O 1s levels in the XPS spectrum of Ni1Co2-S clearly shifted to more positive levels, which was mainly due to electronic interactions involving $\mathrm{S}$ and $\mathrm{Ni} / \mathrm{Co}$ components. ${ }^{25,48}$

\subsection{Electrochemical capacitive behaviours of various electrodes}

To select an efficient hydroxide precursor, we investigated the performances of Ni1Co2, Ni1Co2-AN, Ni3, Ni2Co1 and Co3 in an alkaline three-electrode system. Fig. 5a shows the $C V$ curves of Ni1Co2, Ni1Co2-AN, Ni3, Ni2Co1 and Co3 at $5 \mathrm{mV} \mathrm{s}^{-1}$. The main contents of Ni1Co2, Ni1Co2-AN, Ni3, Ni2Co1 and Co3 were unitary Ni/Co or binary Ni-Co hydroxides. The energy storage mechanism could be described as follows: ${ }^{49-54}$

$$
\begin{gathered}
\mathrm{Ni}(\mathrm{OH})_{2}+\mathrm{OH}^{-} \leftrightarrow \mathrm{NiOOH}+\mathrm{e}^{-} \\
\mathrm{Co}(\mathrm{OH})_{2}+\mathrm{OH}^{-} \leftrightarrow \mathrm{CoOOH}+\mathrm{e}^{-} \\
\mathrm{CoOOH}+\mathrm{OH}^{-} \leftrightarrow \mathrm{CoO} 2+\mathrm{e}^{-}
\end{gathered}
$$

A smaller potential disparity $\left(\Delta E_{\mathrm{a}, \mathrm{c}}\right.$, the disparity between the anode and cathode peak positions) is presented in the $C V$ curve of Ni1Co2, suggesting slight electrode polarization. ${ }^{55,56}$ Additionally, Ni1Co2 delivers longer discharge time, as shown in Fig. 5b, and better capacitance performance, as shown in Fig. 5c, thus highlighting the enhanced performance of the Ni1Co2 electrode. These results are mainly ascribed to the ultrathin flowery structure, providing more active contact sites for charge transfer. In the EIS spectra (Fig. 5d), the intersections of semicircles on the real axis, semicircle diameter at high frequency and slope of a line in the low-frequency region are employed to evaluate the equivalent series resistance $\left(R_{\mathrm{S}}\right)$,
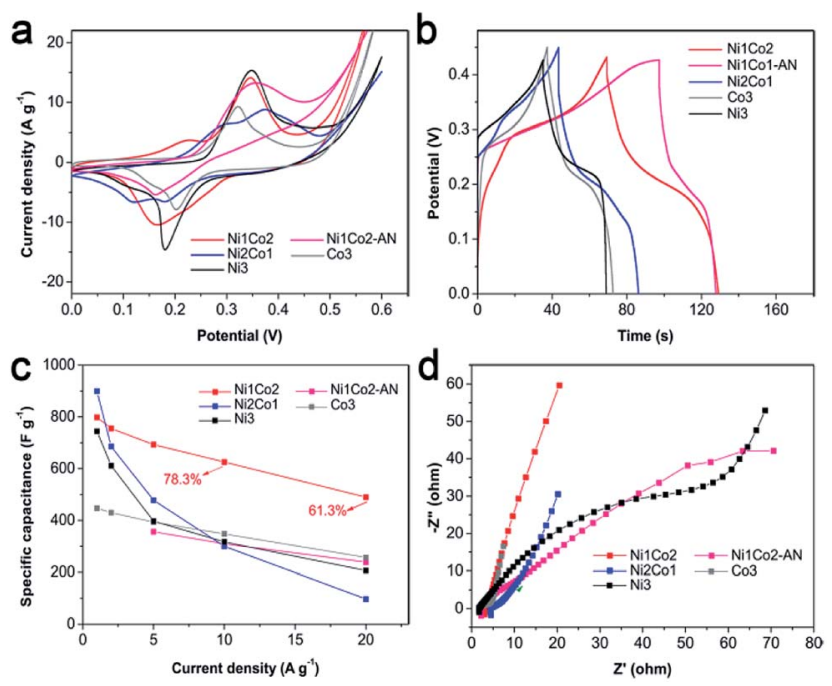

Fig. 5 (a) $C V$ curves at $5 \mathrm{mV} \mathrm{s}^{-1}$, (b) GCD curves at $5 \mathrm{~A} \mathrm{~g}^{-1}$, (c) specific capacitances at various current densities and (d) EIS spectra of $\mathrm{Ni1Co}$, Ni1Co2-AN, Ni2Co1, Co3 and Ni3 electrodes. charge-transfer resistance $\left(R_{\mathrm{ct}}\right)$ and diffusion resistance $\left(Z_{\mathrm{w}}\right) \cdot{ }^{57,58}$ The $R_{\mathrm{S}}$ values of Ni1Co2, Ni1Co2-AN, Ni2Co1, Co3 and Ni3 electrodes are $2.90,2.05,4.38,1.74$ and $1.65 \Omega$, respectively. In comparison, even though Ni1Co2 possesses higher $R_{\mathrm{s}}$ values, the smaller semicircle diameter and steeper slope indicate lower $R_{\mathrm{ct}}$ and $Z_{\mathrm{w}}$ values, thus revealing enhanced performance.

To further promote the performance of Ni1Co2, controllable $\mathrm{S}^{2-}$ grafting was carried out to obtain Ni1Co2-S. The $C V$ curves of Ni1Co2 and Ni1Co2-S at various scanning rates from 5 to $50 \mathrm{mV} \mathrm{s}^{-1}$ are presented in Fig. 6a-c. Compared to that of Ni1Co2, the extended $C V$ area of Ni1Co2-S revealed enhanced capacitance. Interestingly, compared to the observations for Ni1Co2, there was a pair of anodic peaks that disappeared for Ni1Co2-S electrode. This peak disappearance actually resulted due to closer location of various pairs of oxidation and reduction peaks, leading to overlapping. ${ }^{59,60}$ In the $C V$ curves of
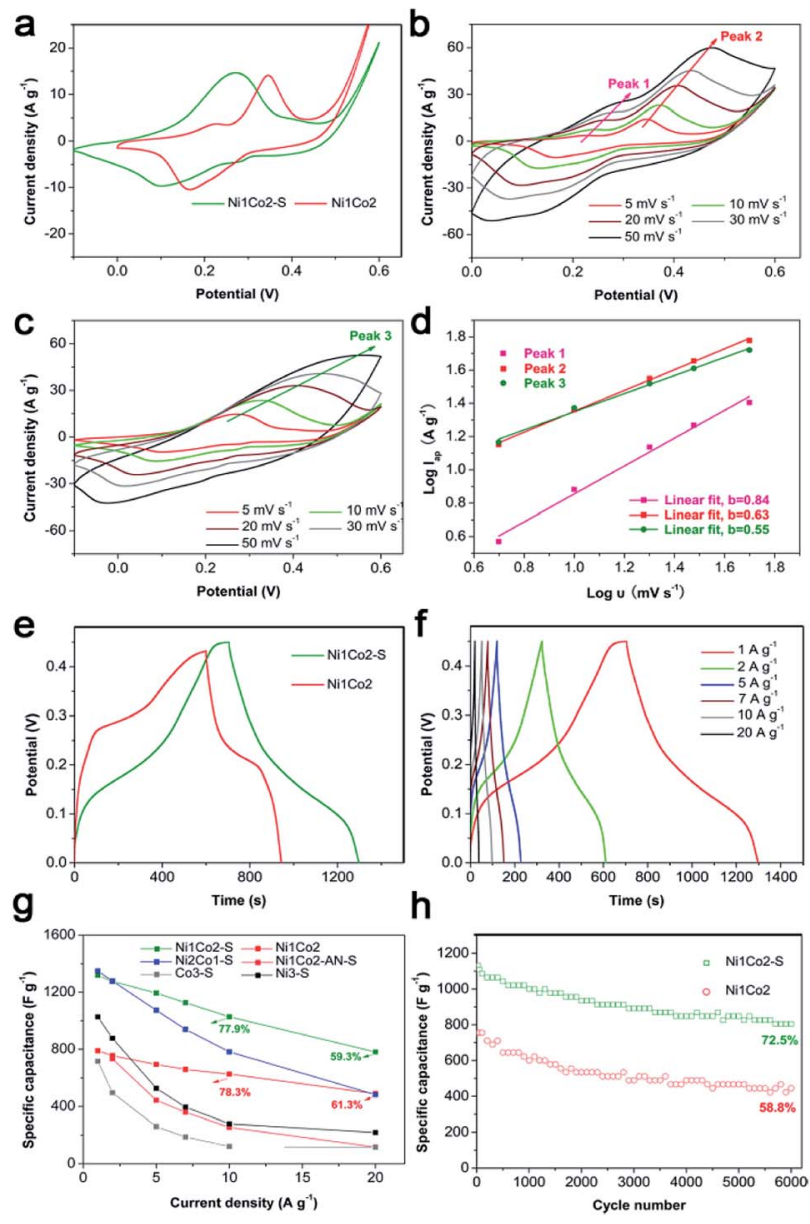

Fig. 6 (a) $\mathrm{CV}$ curves of $\mathrm{Ni1Co} 2$ and Ni1Co2-S at $5 \mathrm{mV} \mathrm{s}^{-1}$; (b) $\mathrm{CV}$ curves of Ni1Co2 at different scanning rates; (c) CV curves of Ni1Co2$S$ at different scanning rates; (d) linear fit of $\log l_{a p}-\log \nu$ scatters from three pairs of peaks in the CV curves of Ni1Co2 and Ni1Co2-S; (e) GCD curves of Ni1Co2 and Ni1Co2-S at $1 \mathrm{~A} \mathrm{~g}^{-1}$; (f) GCD curves of the asfabricated Ni1Co2-S at different current densities; (g) capacitance performances of Ni1Co2, Ni1Co2-S, Ni1Co2-AN-S, Ni2Co1-S, Co3-S and Ni3-S electrodes at various current densities; (h) 6000 cycle duration measurements of $\mathrm{Ni1CO} 2$ and $\mathrm{Ni1CO2}-\mathrm{S}$ at the current density of $10 \mathrm{~A} \mathrm{~g}^{-1}$. 
Ni2Co1-S (Fig. S9a†), there were many pairs of oxidation and reduction peaks, indicating incomplete overlapping of these peaks. Furthermore, the scatters of $\log I_{\mathrm{p}}-\log \nu$ from the three pairs of oxidation peaks are fitted in Fig. 6d, wherein $I_{\mathrm{p}}$ and $\nu$ stand for scanning rate and peak current, respectively. Based on a previous study, the relationship between $I_{\mathrm{p}}$ and $\nu$ is in line with the formula $I=a \nu^{b}$, wherein $a$ and $b$ represent empirical parameters; $b$ value of 0.5 is due to the diffusion-dominated reaction, whereas $b$ value of 1 originates from the ideal capacitive behaviour. ${ }^{55,61}$ In our study, the peak 1 and peak 2 from Ni1Co2 exhibited higher $b$ values of 0.63 and 0.84 , respectively, and the peak 3 from Ni1Co2-S exhibited a lower $b$ value of 0.55 , indicating that the capacitances of Ni1Co2 and Ni1Co2-S contained diffusion and capacitive contributions.

As shown in Fig. 6e and f, Ni1Co2-S displayed low discharge platform, which was in agreement with the result of $C V$ curves. We further calculated the specific capacitances of our electrodes based on different GCD curves from 1 to $20 \mathrm{~A} \mathrm{~g}^{-1}$. As shown in Fig. 6g, Ni1Co2-S possessed enhanced specific capacitances (1317.8 $\mathrm{F} \mathrm{g}^{-1}$ at $1 \mathrm{~A} \mathrm{~g}^{-1}$ and $781.3 \mathrm{~F} \mathrm{~g}^{-1}$ at $20 \mathrm{~A} \mathrm{~g}^{-1}$ ) relative to those of $\mathrm{Ni1Co} 2\left(789.5 \mathrm{~F} \mathrm{~g}^{-1}\right.$ at $1 \mathrm{~A} \mathrm{~g}^{-1}$ and $489.4 \mathrm{~F} \mathrm{~g}^{-1}$ at $20 \mathrm{~A} \mathrm{~g}^{-1}$ ). When the Ni1Co2-S electrode was at high current density of 10 or $20 \mathrm{~A} \mathrm{~g}^{-1}$, it still displayed suitable capacitance retention of $77.9 \%$ or $59.3 \%$, respectively. The electrochemical capacitances of Ni1Co2-AN-S, Ni2Co1-S, Ni3-S and Co3-S were also studied. Similarly, $\mathrm{S}^{2-}$ ion grating enhanced the capacitance compared with that of the corresponding precursors. Ni1Co2-S displayed excellent capacitance and high rate performance. Therefore, the selection of precursor and further $\mathrm{S}^{2-}$ ion grating efficiently promoted the comprehensive performances of our electrode materials.

Besides, long-term cycling duration is desired for reversible applications; hence, the cycling measurement of Ni1Co2-S was operated at a current density of $10 \mathrm{~A} \mathrm{~g}^{-1}$. In Fig. 6h, after 6000 cycles, Ni1Co2-S presented a high capacitance of $804.3 \mathrm{~F} \mathrm{~g}^{-1}$ and capacitance retention of $72.5 \%$, which was higher than that of Ni1Co2 $(58.8 \%)$. As shown in Fig. 7, Ni1Co2-S had lower semicircle diameter and steeper slope (lower $R_{\mathrm{ct}}$ and $Z_{\mathrm{w}}$ ), further highlighting the improved resistance performance. $R_{\mathrm{ct}}$ and $Z_{\mathrm{w}}$ significantly increased after 6000 cycles, probably due to the damage of internal nanosheets of Ni1Co2-S (in Fig. S10 $\dagger$ ). In view of the results mentioned above, the enhancement in the
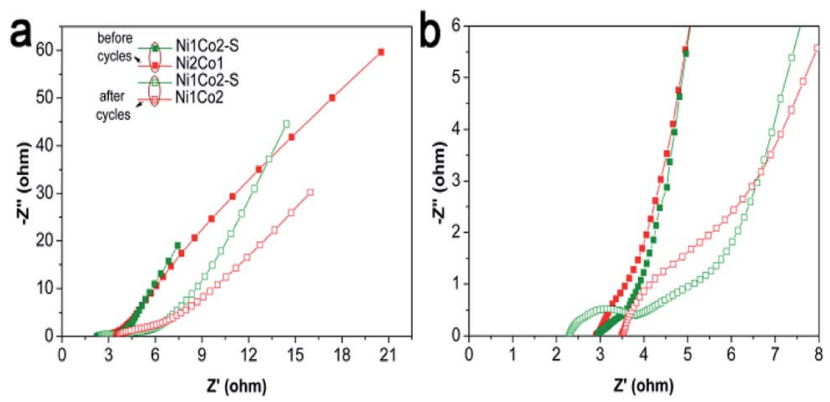

Fig. 7 (a) EIS spectra of Ni1Co2 and Ni1Co2-S electrodes before and after 6000 cycles with frequencies from 0.01 to $100 \mathrm{kHz}$; (b) magnification of EIS spectra from 0 to $8 \Omega$ for $Z^{\prime}$. electrochemical performance of Ni1Co2-S was mainly due to the following factors: (1) suitable ratio of $\mathrm{Ni}$ : Co could tune the nanostructure and promote electrochemical performance; (2) S was introduced into $\mathrm{Ni}$-Co hydroxide, and it greatly improved the conductivity; and (3) $\mathrm{S}^{2-}$ ion grafting generated many defects and extended the pore size.

\subsection{Electrochemical capacitive behaviour of HSC device}

Considering that general three-electrode systems have narrow potential windows, we prepared an HSC device to enlarge the voltage using Ni1Co2-S and AC electrodes as positive and negative electrodes, respectively, as shown in Fig. 8a. The $C V$ curves shown in Fig. 8b present large quasi-rectangular shapes with few weak redox peaks, which suggest the battery-type and electrical double-layer hybrid energy-storage mechanisms. Fig. 8c displays various linear GCD profiles with long discharge time, further highlighting its good capacitance performance. As depicted in Fig. 8d, Ni1Co2-S//AC delivered great specific capacitance values of 117.1, 106.3, 97.5, 82.3, 74.5 and $61.1 \mathrm{~F}$ $\mathrm{g}^{-1}$ at various current densities of $1,2,3,5,7$ and $10 \mathrm{~A} \mathrm{~g}^{-1}$, respectively. Unfortunately, with the increase in current density, the specific capacity gradually faded, and the total voltage of the HSC also increased due to insufficient electrochemical reaction at high current density. Similar to the study of the threeelectrode system, we also studied the cycling stability of Ni1Co2-S//AC with charge/discharge current density of $3 \mathrm{~A} \mathrm{~g}^{-1}$ (in Fig. 8e). The initial electrochemical measurement contributed to gradual wetting between the electrode and electrolyte. The trapped $\mathrm{OH}^{-}$ions then gradually diffused out, and the contact area between the electrode and electrolyte was enlarged,

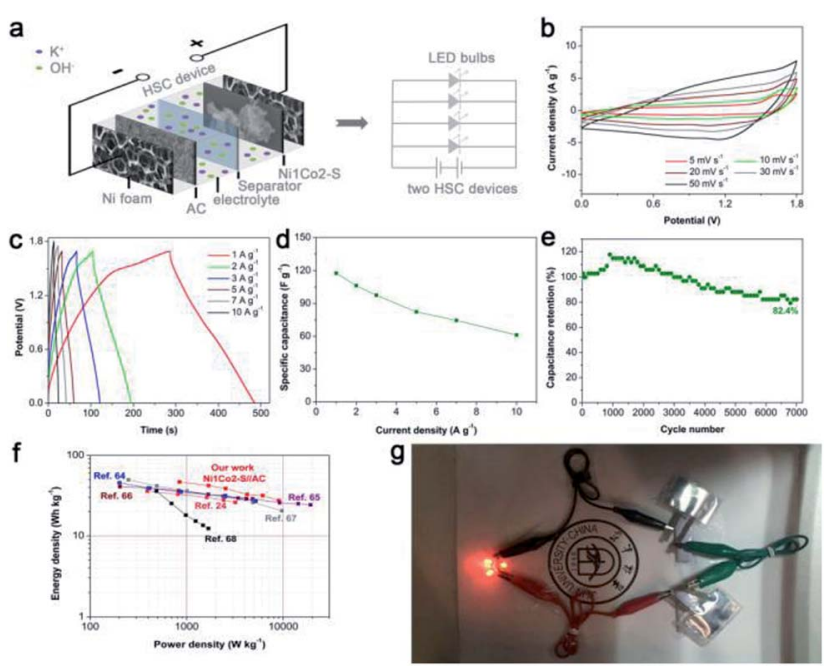

Fig. 8 (a) Schematic diagram of Ni1Co2-S//AC and lighting LED bulb devices; (b) CV curves of the as-fabricated Ni1Co2-S//AC at different scanning rates from 5 to $50 \mathrm{mV} \mathrm{s}^{-1}$; (c) GCD curves of Ni1Co2-S//AC device at various current densities from 1 to $10 \mathrm{~A} \mathrm{~g}^{-1}$; (d) specific capacitance of Ni1CO2-S//AC device at various current densities from 1 to $10 \mathrm{~A} \mathrm{~g}^{-1}$; (e) cycling performance of Ni1Co2-S//AC for 7000 cycles at $3 \mathrm{~A} \mathrm{~g} \mathrm{~g}^{-1}$; (f) Ragone plots of our study and other peer HSC devices; and ( $\mathrm{g}$ ) photographs of lighting LED bulbs device with fast charging current of $3 \mathrm{~A} \mathrm{~g}^{-1}$ based on total mass of active materials. 
thus causing capacitance enhancement during the initial 1000 cycles. ${ }^{6,63}$ Subsequently, the capacitance of Ni1Co2-S//AC slowly faded and reached $82.4 \%$ after 7000 cycles at $3 \mathrm{~A} \mathrm{~g}^{-1}$, resulting from partial damage to the Ni1Co2-S structure.

The relationship between energy density and power density in Ni1Co2-S//AC is depicted in the Ragone diagram (Fig. 8f). The HSC device achieved a high energy density of $46.7 \mathrm{~W} \mathrm{~h} \mathrm{kg^{-1 }}$ at a power density of $0.845 \mathrm{~kW} \mathrm{~kg}^{-1}$ and also reached $27.5 \mathrm{~W} \mathrm{~h}$ $\mathrm{kg}$ at $9 \mathrm{~kW} \mathrm{~kg}^{-1}$, which were comparable to the results of many peer HSC devices based on core-shell $\mathrm{NiCo}_{2} \mathrm{~S}_{4} @ \mathrm{Co}(\mathrm{OH})_{2}$ nanotube arrays, ${ }^{24} \mathrm{NiCo}_{2} \mathrm{~S}_{4}$ nanosheets, ${ }^{64}$ and other cobalt/ nickel hydroxide electrode materials. ${ }^{65-68}$ To trigger four light emitting diodes (LEDs, red, $5 \mathrm{~mm}$ ), we further assembled two Ni1Co2-S//AC devices in series and charged them at $3 \mathrm{Ag}^{-1}$, as exhibited in Fig. 6g. As anticipated, the LED bulbs worked for a long duration of $2 \mathrm{~min}$ and displayed dim light after $5 \mathrm{~min}$. These results suggested that Ni1Co2-S can serve as a promising energy storage material.

\section{Conclusions}

Herein, we designed a simple solid-liquid interface reaction to synthesize Ni1Co2-S using $\mathrm{NaOH} /$ agar and $\mathrm{Na}_{2} \mathrm{~S}$ /agar hydrogels. Ni1Co2-S was mainly composed of $\alpha$-(Ni-Co) hydroxide with $S^{2-}$ ion grating. The slight ion exchange reaction between $\mathrm{S}^{2-}$ ions and Ni1Co2 introduced a small amount of sulphide to improve its conductivity and expanded the internal pore size, thus ensuring rapid charge transfer. Compared to other Ni1Co2, Ni1Co2-AN, Ni2Co1, Co3 and Ni3 electrodes, the Ni1Co2-S electrode delivered improved capacitance of $1317.8 \mathrm{~F}$ $\mathrm{g}^{-1}$ at $1 \mathrm{~A} \mathrm{~g}^{-1}$, suitable rate performance $\left(77.9 \%\right.$ at $10 \mathrm{~A} \mathrm{~g}^{-1}$ and $59.3 \%$ at $20 \mathrm{~A} \mathrm{~g}^{-1}$ ) and improved cycling stability. The Ni1Co2S//AC device also exhibited a high energy density of 46.7 $\mathrm{W} \mathrm{h} \mathrm{kg}^{-1}$ at $0.845 \mathrm{~W} \mathrm{~kg}^{-1}$ and long cycling stability, which further demonstrated the potential advantage of Ni1Co2-S material in supercapacitive storage.

\section{Conflicts of interest}

There are no conflicts to declare.

\section{Acknowledgements}

This work was financially supported by the National Natural Science Foundation of China (Grant No. 51501068) and Graduate Innovation Fund of Jilin University (Grant No. 2017183).

\section{Notes and references}

1 K. M. Hercule, Q. Wei, A. M. Khan, Y. Zhao, X. Tian and L. Mai, Nano Lett., 2013, 13, 5685-5691.

2 P. Simon and Y. Gogotsi, Nat. Mater., 2008, 7, 845-854. 3 X. Zhang, Y. Zhao and C. Xu, Nanoscale, 2014, 6, 3638-3646. 4 T. Kim, G. Jung, S. Yoo, K. S. Suh and R. S. Ruoff, ACS Nano, 2013, 7, 6899-6905.

5 B. Y. Guan, L. Yu, X. Wang, S. Song and X. W. Lou, Adv. Mater., 2017, 29, 1605051.
6 J. H. Won, H. M. Jeong and J. K. Kang, Adv. Energy Mater., 2017, 7, 1601355.

7 T. Brousse, D. Belanger and J. W. Long, J. Electrochem. Soc., 2015, 162, A5185-A5189.

8 P. Simon, Y. Gogotsi and B. Dunn, Science, 2014, 343, 12101211.

9 Y. Gogotsi, ACS Nano, 2014, 8, 5369-5371.

10 D. Zhou, X. Su, M. Boese, R. Wang and H. Zhang, Nano Energy, 2014, 5, 52-59.

11 L. Wang, Z. H. Dong, Z. G. Wang, F. X. Zhang and J. Jin, Adv. Funct. Mater., 2013, 23, 2758-2764.

12 J. Ji, L. L. Zhang, H. Ji, Y. Li, X. Zhao, X. Bai, X. Fan, F. Zhang and R. S. Ruoff, ACS Nano, 2013, 7, 6237-6243.

13 R. B. Rakhi, W. Chen, D. Cha and H. N. Alshareef, Nano Lett., 2012, 12, 2559-2567.

14 V. R. Shinde, S. B. Mahadik, T. P. Gujar and C. D. Lokhande, Appl. Surf. Sci., 2006, 252, 7487-7492.

15 M. Liu, L. Gan, W. Xiong, Z. Xu, D. Zhu and L. Chen, J. Mater. Chem. A, 2014, 2, 2555-2562.

16 K. Ramasamy, R. K. Gupta, S. Palchoudhury, S. Ivanov and A. Gupta, Chem. Mater., 2015, 27, 379-386.

17 M. Chauhan, K. P. Reddy, C. S. Gopinath and S. Deka, ACS Catal., 2017, 7, 5871-5879.

18 X. Chen, Y. Huang, K. Zhang, X. Feng and S. Li, J. Alloys Compd., 2016, 686, 905-913.

19 G. C. Lau, N. A. Sather, H. Sai, E. M. Waring, E. Deiss-Yehiely, L. Barreda, E. A. Beeman, L. C. Palmer and S. I. Stupp, Adv. Funct. Mater., 2018, 28, 1702320.

20 Y. Liu, N. Fu, G. Zhang, M. Xu, W. Lu, L. Zhou and H. Huang, Adv. Funct. Mater., 2017, 27, 1605307.

21 J. H. Park, O. O. Park, K. H. Shin, C. S. Jin and J. H. Kim, Electrochem. Solid-State Lett., 2002, 5, H7-H10.

22 D. Lee, Q. X. Xia, J. M. Yun and K. H. Kim, Appl. Surf. Sci., 2018, 433, 16-26.

23 W. Zhou, X. Cao, Z. Zeng, W. Shi, Y. Zhu, Q. Yan, H. Liu, J. Wang and H. Zhang, Energy Environ. Sci., 2013, 6, 22162221.

24 R. Li, S. Wang, Z. Huang, F. Lu and T. He, J. Power Sources, 2016, 312, 156-164.

25 X. Zhu, X. Dou, J. Dai, X. An, Y. Guo, L. Zhang, S. Tao, J. Zhao, W. Chu, X. C. Zeng, C. Wu and Y. Xie, Angew. Chem., Int. Ed., 2016, 55, 12465-12469.

26 T. Yoon and K. S. Kim, Adv. Funct. Mater., 2016, 26, 73867393.

27 T. Yan, R. Li, L. Zhou, C. Ma and Z. Li, Electrochim. Acta, 2015, 176, 1153-1164.

28 Y. Lu, Y. Wang, Y. Zou, Z. Jiao, B. Zhao, Y. He and M. Wu, Electrochem. Commun., 2010, 12, 101-105.

29 F.-X. Ma, H. Hu, H. B. Wu, C.-Y. Xu, Z. Xu, L. Zhen and X. W. Lou, Adv. Mater., 2015, 27, 4097-4101.

30 S. Ratha, A. K. Samantara, K. K. Singha, A. S. Gangan, B. Chakraborty, B. K. Jena and C. S. Rout, ACS Appl. Mater. Interfaces, 2017, 9, 9640-9653.

31 F. Grote, Z.-Y. Yu, J.-L. Wang, S.-H. Yu and Y. Lei, Small, 2015, 11, 4666-4672.

32 B. Li, X. Li, J. Zai and X. Qian, Nano-Micro Lett., 2016, 8, 174181. 
33 M. Rajamathi, P. V. Kamath and R. Seshadri, J. Mater. Chem., 2000, 10, 503-506.

34 P. V. Kamath, G. H. A. Therese and J. Gopalakrishnan, J. Solid State Chem., 1997, 128, 38-41.

35 M. Rajamathi, G. N. Subbanna and P. V. Kamath, J. Mater. Chem., 1997, 7, 2293-2296.

36 T. Peng, H. Wang, H. Yi, Y. Jing, P. Sun and X. Wang, Electrochim. Acta, 2015, 176, 77-85.

37 O. Bockman, T. Ostvold, G. A. Voyiatzis and G. N. Papatheodorou, Hydrometallurgy, 2000, 55, 93-105.

38 Y. Wang, H. Wang and X. Wang, Electrochim. Acta, 2013, 92, 298-303.

39 W. Tian, X. Wang, C. Zhi, T. Zhai, D. Liu, C. Zhang, D. Golberg and Y. Bando, Nano Energy, 2013, 2, 754-763.

40 X. Xiong, D. Ding, D. Chen, G. Waller, Y. Bu, Z. Wang and M. Liu, Nano Energy, 2015, 11, 154-161.

41 J. Yan, Z. Fan, W. Sun, G. Ning, T. Wei, Q. Zhang, R. Zhang, L. Zhi and F. Wei, Adv. Funct. Mater., 2012, 22, 2632-2641.

42 C. Hou, X.-Y. Lang, Z. Wen, Y.-F. Zhu, M. Zhao, J.-C. Li, W.-T. Zheng, J.-S. Lian and Q. Jiang, J. Mater. Chem. A, 2015, 3, 23412-23419.

43 J. Liang, R. Ma, N. Iyi, Y. Ebina, K. Takada and T. Sasaki, Chem. Mater., 2010, 22, 371-378.

$44 \mathrm{H}$. Chen, L. Hu, M. Chen, Y. Yan and L. Wu, Adv. Funct. Mater., 2014, 24, 934-942.

45 W. Du, Z. Wang, Z. Zhu, S. Hu, X. Zhu, Y. Shi, H. Pang and X. Qian, J. Mater. Chem. A, 2014, 2, 9613-9619.

46 L. Niu, Y. Wang, F. Ruan, C. Shen, S. Shan, M. Xu, Z. Sun, C. Li, X. Liu and Y. Gong, J. Mater. Chem. A, 2016, 4, 56695677.

47 T. Li, Y. Zuo, X. Lei, N. Li, J. Liu and H. Han, J. Mater. Chem. A, 2016, 4, 8029-8040.

48 J.-X. Feng, H. Xu, Y.-T. Dong, S.-H. Ye, Y.-X. Tong and G.-R. Li, Angew. Chem., Int. Ed., 2016, 55, 3694-3698.

49 H. Ma, J. He, D.-B. Xiong, J. Wu, Q. Li, V. Dravid and Y. Zhao, ACS Appl. Mater. Interfaces, 2016, 8, 1992-2000.

50 Y. Zhao, H. Ma, S. Huang, X. Zhang, M. Xia, Y. Tang and Z.-F. Ma, ACS Appl. Mater. Interfaces, 2016, 8, 22997-23005.
51 M. Wang, F. Jin, X. Zhang, J. Wang, S. Huang, X. Zhang, S. Mu, Y. Zhao and Y. Zhao, ACS Sustainable Chem. Eng., 2017, 5, 5679-5685.

52 Y. Zhao, Z. Chen, D.-B. Xiong, Y. Qiao, Y. Tang and F. Gao, Sci. Rep., 2016, 6, 17613.

53 Y. Zhao, X. Zhang, J. He, L. Zhang, M. Xia and F. Gao, Electrochim. Acta, 2015, 174, 51-56.

54 Z. Chen, D.-B. Xiong, X. Zhang, H. Ma, M. Xia and Y. Zhao, Nanoscale, 2016, 8, 6636-6645.

55 J. Liu, M. Chen, L. Zhang, J. Jiang, J. Yan, Y. Huang, J. Lin, H. J. Fan and Z. X. Shen, Nano Lett., 2014, 14, 7180-7187.

56 J. Liu, J. Wang, Z. Ku, H. Wang, S. Chen, L. Zhang, J. Lin and Z. X. Shen, ACS Nano, 2016, 10, 1007-1016.

57 Y. Zeng, Y. Han, Y. Zhao, Y. Zeng, M. Yu, Y. Liu, H. Tang, Y. Tong and X. Lu, Adv. Energy Mater., 2015, 5, 1402176.

58 L.-L. Zhang, H.-H. Li, C.-Y. Fan, K. Wang, X.-L. Wu, H.-Z. Sun and J.-P. Zhang, J. Mater. Chem. A, 2015, 3, 19077-19084.

59 Q. Liao, N. Li, S. Jin, G. Yang and C. Wang, ACS Nano, 2015, 9, 5310-5317.

60 Y. Cheng, H. Zhang, C. V. Varanasi and J. Liu, Energy Environ. Sci., 2013, 6, 3314-3321.

61 X. Xu, J. Liu, Z. Liu, J. Shen, R. Hu, J.-W. Liu, L. Ouyang, L. Zhang and M. Zhu, ACS Nano, 2017, 11, 9033-9040.

62 J. Chang, M. Jin, F. Yao, T. H. Kim, L. Viet Thong, H. Yue, F. Gunes, B. Li, A. Ghosh, S. Xie and Y. H. Lee, Adv. Funct. Mater., 2013, 23, 5074-5083.

63 S. Saha, M. Jana, P. Khanra, P. Samanta, H. Koo, N. C. Murmu and T. Kuila, RSC Adv., 2016, 6, 1380-1387.

64 L. Shen, J. Wang, G. Xu, H. Li, H. Dou and X. Zhang, Adv. Energy Mater., 2015, 5, 1400977.

65 J.-C. Chen, C.-T. Hsu and C.-C. Hu, J. Power Sources, 2014, 253, 205-213.

66 Y. Cheng, H. Zhang, C. V. Varanasi and J. Liu, Energy Environ. Sci., 2013, 6, 3314-3321.

67 H. Jiang, C. Li, T. Sun and J. Ma, Chem. Commun., 2012, 48, 2606-2608.

68 H. B. Li, M. H. Yu, F. X. Wang, P. Liu, Y. Liang, J. Xiao, C. X. Wang, Y. X. Tong and G. W. Yang, Nat. Commun., 2013, 4, 1894. 